\title{
DIPLOMAZIA INFORMALE E STRATEGIE DI RESILIENZA. IL MATRIMONIO FRA CARLO EMANUELE II DI SAVOIA E MADEMOISELLE DE VALOIS NELLE LETTERE DI MARGHERITA DI LORENA, DUCHESSA D'ORLÉANS, A CRISTINA DI FRANCIA
}

\author{
Elisabetta Lurgo \\ (Università Ca' Foscari Venezia) \\ elisabetta.lurgo@unive.it
}

\section{RIASUNTO}

Il presente articolo propone un'analisi della corrispondenza fra Margherita di Lorena, vedova di Gastone d'Orléans (1615-1672) e Cristina di Francia, vedova del duca Vittorio Amedeo I di Savoia, in occasione delle negoziazioni per le nozze tra la figlia di Margherita, Mademoiselle di Valois, e Carlo Emanuele II, duca di Savoia. L'obiettivo è quello di mostrare le opportunità offerte da una multicentrica società di corte a una principessa dallo statuto controverso, per sviluppare una forma di resilienza ed esercitare un'influenza informale sulle strategie matrimoniali di una dinastia regnante.

PAROLE CHIAVE: Storia della Francia in età moderna; Stati Sabaudi; Diplomazia informale; Studi sulle Corti; Corrispondenza.

\section{INFORMAL DIPLOMACY AND STRATEGIES OF RESILIENCE. THE MARRIAGE BETWEEN CHARLES EMMANUEL II OF SAVOY AND MADEMOISELLE DE VALOIS IN THE LETTERS BY MARGUERITE OF LORRAINE, DUCHESS OF ORLÉANS, TO CHRISTINE DE FRANCE}

\begin{abstract}
This article means to analyze the correspondence between Margaret of Lorraine, widow of Gaston d'Orléans (1615-1672), and Christine of France, widow of Vittorio Amedeo I of Savoy, on the occasion of the marriage negotiations between the daughter of Margherita, Mademoiselle of Valois, and Carlo Emanuele II, Duke of Savoy. The aim is to show the opportunities that a multicentric court society offers to a Princess enjoying very high yet controversial status, to develop a strategy of resilience and to exercise an informal influence on matrimonial politics of a ruling dynasty.
\end{abstract}


KEY WORDS: Early Modern France; States of Savoy; Informal Diplomacy; Court Studies; Correspondences.

\section{INTRODUZIONE}

Il 4 marzo 1663, nella cappella del Louvre, il cardinale Antonio Barberini, grande elemosiniere di Luigi XIV, celebrava le nozze tra Francesca Maddalena d'Orléans, figlia di Gastone d'Orléans, fratello di Luigi XIII, e Carlo Emanuele II, duca di Savoia ${ }^{1}$. Da parte sabauda, i negoziati ufficiali furono affidati all'abate Girolamo Amoretti, elemosiniere di Cristina di Francia, duchessa vedova di Savoia, e al marchese Ghiron Francesco Villa, ambasciatore straordinario a Parigi ${ }^{2}$. Ma promotrici dell'alleanza furono in primo luogo le madri dei due giovani sposi: Cristina di Francia, detta Madama Reale, saldamente al governo dello Stato sabaudo nonostante la maggiore età del figlio, e Margherita di Lorena, seconda moglie di Gastone ${ }^{3}$. Nei paragrafi seguenti, mi propongo di analizzare il ruolo svolto da quest'ultima nel corso delle trattative matrimoniali con la corte di Torino e i legami da essa intrecciati con Cristina in occasione delle nozze: a questo scopo, utilizzerò come fonte principale le lettere di Margherita a Cristina di Francia ${ }^{4}$.

La corrispondenza di Margherita di Lorena con Madama Reale può essere studiata attraverso tre chiavi di lettura. In primo luogo, le lettere di Margherita, che fino al progetto di matrimonio sabaudo intrattiene con Cristina una corrispondenza puramente di cortesia, ci restituiscono l'immagine di una donna confrontata a un ambiente ostile, abitata dall'ossessione dell'intrigo, ma rivelano anche la

\footnotetext{
${ }^{1}$ Su Carlo Emanuele II (1634-1675) manca una monografia moderna. Cfr. Vittorio Castronovo in Dižionario Biografico degli Italiani, Roma, Treccani, vol. 20, 1977, CARLO EMANUELE II, duca di Savoia in "Dizionario Biografico" (treccani.it); ancora utili i vecchi lavori di Gaudenzio Claretta, Storia del regno di Carlo Emanuele II, (Genova: 1877), e di Ercole Ricotti, Storia della monarchia piemontese (Firenze: Barbera, 1861-1869), vol. VI.

2 Il contratto e le capitolazioni matrimoniali si trovano a Torino, Archivio di Stato [AsTo], Matrimoni Real Casa, m. 30, fasc. 10-11. Sul marchese Villa (1617-1670), si veda Blythe A. Raviola in Dizionario Biografico degli Italiani, vol. 99, 2020, VILLA, Ghiron Francesco in "Dizionario Biografico" (treccani.it).

${ }^{3}$ Su Cristina di Francia, duchessa di Savoia (1606-1663), cfr. Giuliano Ferretti, ed., L'État, la cour et la ville. Le duché de Savoie au temps de Victor-Amédée Ier et de Christine de France, 1618-1663 (Paris: Classiques Garnier, 2018); “Christine de France et son siècle," Revue Dix-septième siècle 262 (2014); G. Ferretti, dir., De Paris à Turin. Christine de France duchesse de Savoie (Paris: L'Harmattan, 2014); Alain Becchia e Florine Vital-Durand, eds. Édifier l'État: politique et culture en Savoie au temps de Christine de France (Chambéry: USMB, 2014). Sulla posizione subalterna di Carlo Emanuele rispetto alla madre, cfr. Kristine Kolrud, “The Prolonged Minority of Charles Emmanuel II”, in Sabaudian Studies. Political Culture, Dynasty and Territory, 1400-1700, ed. Mathew Vester, (Truman State University Press, 2013), 191-209.

${ }_{4}^{4}$ AsTo, Materie politiche per rapporto all'interno, Lettere Principi forestieri, m. 39.
} 
consapevolezza con cui la principessa utilizza il poco margine di manovra concessole, sfruttando le reti di solidarietà e di rivalità su cui si basa il sistema-corte. Nonostante la crescente professionalizzazione degli ambasciatori, infatti, la diplomazia di età moderna rimane a lungo un linguaggio sociale declinato nell'ambito della cosiddetta «società dei principi» ${ }^{5}$ : esso si basa non soltanto sulle rappresentazioni rituali del potere, ma anche sui sistemi clientelari che agiscono, in modo spesso informale, sulle pratiche di governo ${ }^{6}$. In questa prospettiva, la recente storiografia ha rivolto una crescente attenzione al ruolo delle donne, in una società di corte il cui fulcro è un potere dinastico ancorato nella «famiglia», intesa come rete di legami personali ${ }^{7}$. In ambito statunitense, si è proposto di ricorrere alla nozione di soft power, per studiare l'influenza esercitata dalle donne nelle corti europee di età moderna ${ }^{8}$. Tuttavia, l'utilizzo di tale categoria concettuale sottintende l'esistenza di un potere «al femminile», con caratteri propri, prevalentemente relegato alla sfera privata e subordinato, dunque, a un potere più propriamente maschile, istituzionalmente e ritualmente legittimato. Per uscire da

\footnotetext{
${ }^{5}$ Secondo la fortunata espressione di L. Bély, La société des princes, XVI $I^{e} X V I I I^{e}$ siècle, Paris, Fayard, 1999.

${ }^{6}$ Cfr. Jean-Louis Fournel e Matteo Residori, eds. Ambassades et ambassadeurs en Europe (XV'- XVII ${ }^{e}$ siècles) (Genève: Droz, 2020); Stefano Andretta, Lucien Bély e Geraud Poumarède, dir., Esperienza e diplomazia. Saperi, pratiche culturali e ažione diplomatica nell'Età moderna (secc. XV-XVIII) (Roma: Viella, 2020); Indravati Felicité, dir., L'identité du diplomate (Moyen Âge-XIX'e siècle). Métier ou noble loisir? (Paris: Garnier, 2020); Dejanirah Couto e Stéphane Péguinot, dir., Les langues de la négociation. Approches historiennes (Rennes: PUR, 2017); Tracey A. Sowerby e Jan Hennings, eds., Pratices of Diplomacy in the Early Modern World (Routledge, 2017); Stefano Andretta, Stéphane Peguinot e Marie-Karine Schaub, eds., Paroles de négociateurs. L'entretien dans la pratique diplomatique de la fin du Moyen Age à la fin du XIXe siècle (Roma: École Française, 2010.) Sulle pratiche diplomatiche nello Stato sabaudo del Seicento, cfr. Pierpaolo Merlin, $L a$ croce e il giglio. Il ducato di Savoia e la Francia tra XVI e XVII secolo (Roma: Carocci, 2018); Alessandro Celi e Mathew Vester, eds., Tra Francia e Spagna. Reti diplomatiche, territori e culture nei domini sabaudi fra Tre e Settecento (Roma: Carocci, 2017); Andrea Pennini, "Con la massima diligentia possible". Diplomazia e politica estera sabauda nel primo Seicento (Roma: Carocci, 2015); Toby Osborne, Dynasty and Diplomacy in the Court of Savoy: Political Culture and the Thirty Years War (Cambridge: CUP, 2000); Christopher Storrs, "Savoyard Diplomacy in the Eighteen Century (1684-1798)" in Politics and Diplomacy in Early Modern Italy. The Structure of Diplomatic Practice, 1450-1800 (Cambridge: CUP, 2000), 210-53; Idem, War, Diplomacy and the Rise of Savoy, 1690-1720 (Cambridge: CUP, 1999).

${ }^{7}$ Cfr. Jeroen Duindam, "The Court as a Meeting Point: Cohesion, Competition, Control”, in Prince, Pen and Sword: Eurasian Perspectives, ed. Maaike van Berkel e Jeroen Duindam (Leiden-Boston: Brill, 2018), 32-128; Rubén Gonzalez Cuerva e Alexander Koller, dir., A Europe of Courts, a Europe of Factions: Political Groups at Early Modern Centres of Power (1550-1700), (Brill, 2017); Jeroen Duindam, "Pre-modern Power Elites: Princes, Courts, Intermediaries" in The Palgrave Handbook of Political Elites (Palgrave, 2017), 161 179; Nadine Akkerman e Birgit Houben, eds., The Politics of Female Households: Ladies-in-waiting across Early Modern Europe (Leiden, Brill, 2014); Dena Goodman, "Public Sphere and Private Life: Toward a Synthesis of Current Historiographical Approaches to the Old Regime," History and Theory 31 (1992): 120.

8 Anne Twomey, "The Exercise of Soft Power by Female Monarchs in the United Kingdom," Royal Studies Journal 7 (2020): 31-48; Frank Muller e Heidi Mehrkens, eds. Royal Heirs and the Uses of Soft Power in Nineteenth-Century Europe (Palgrave, 2016). Joseph S. Nye, "Public Diplomacy and Soft Power," The Annal of American Academy of Political and Social Science 616 (2008): 94-109, ha definito il soft power come "the ability to affect others to obtain the outcomes one wants through attraction rather than coercion or payment» (ibid., 94).
} 
un'impasse che finisce per riprodurre stereotipi di genere nel momento stesso in cui propone di superarli, il concetto di network può rivelarsi, invece, decisivo: erano le reti di relazioni, infatti, a consentire agli individui di costruirsi ambiti di influenza informale, aldilà dei ruoli istituzionali, creando uno spazio "polifonico» di contrattazione del potere, che coinvolge tanto figure maschili quanto femminili'.

In secondo luogo, le lettere di Margherita di Lorena a Cristina di Francia gettano luce su quel reticolo di agenti informali e di vere e proprie spie, sparsi nelle corti europee, che costituiscono una vera e propria miniera di informazioni per principi e ministri ${ }^{10}$. Infine, esse possono essere lette in un'altra prospettiva: vale a dire, come una strategia di resilienza elaborata da chi, pur occupando un rango teoricamente molto elevato, vive in realtà una condizione di marginalità all'interno della gerarchia di corte $^{11}$. Da un lato, infatti, Margherita cerca di esercitare un'influenza per così dire occulta sulla strategia matrimoniale di due dinastie regnanti, quella francese e quella sabauda; dall'altro lato, la principessa tenta di compensare un'effettiva marginalità elaborando un linguaggio «resiliente», attraverso l'uso ridondante del registro affettivo, ben aldilà delle convenzioni retoriche imposte dagli scambi epistolari ${ }^{12}$.

Per comprendere la condotta di Margherita in appoggio all'alleanza con il duca di Savoia e il suo approccio verso Cristina di Francia e Carlo Emanuele, occorre ricordare il suo status peculiare all'interno della famiglia reale. Figlia di Francesco di Lorena, conte di Vaudémont, la principessa aveva sposato il duca d'Orléans, allora erede presuntivo al trono di Francia, nel 1632. La cerimonia si era tenuta segretamente a Nancy, con l'avallo del fratello di Margherita, Carlo IV, duca di Lorena, ma senza chiedere il preventivo consenso di Luigi XIII: per questo motivo, la validità del

${ }^{9}$ Cfr. Réseaux de femmes, femmes en réseaux (XVI'-XVIII siècles) (Bordeaux: Presses Universitaires de Bordeaux, 2018); Marcella Aglietti e José A. López Anguita, eds., Élites internazionali e reti di potere (Pisa: Pisa University Press, 2016), in particolare il contributo, di Elena Riva, "La crisi dinastica e le reti di potere cortigiane nei dispacci degli ambasciatori sabaudi a Madrid. Alcune linee di lettura", 199-213; Diana Carrió-Invernizzi, "A New Diplomatic History and the Networks of Spanish Diplomacy in the Baroque Era," The International History Review 36 (2014): 603-618; Anna Blum, La diplomatie de la France en Italie du Nord au temps de Richelieu et de Mazarin (Paris: Garnier, 2014), 449-485; Elena Riva, "Women, Power and Culture in the European Transition between the XVII ${ }^{\text {th }}$ and the XVIII ${ }^{\text {th }}$ century", in The Transition in Europe between XVII th and XVIII th centuries. Perspectives and Case Studies, ed. Antonio ÁlvarezOssorio, Cinzia Cremonini e Elena Riva (Milano: Franco Angeli, 2016), 62-70.

${ }^{10}$ Cfr. Lucien Bély, Les Secrets de Louis XIV. Mystères d'État et pouvoir absolu (Paris: Tallandier, 2015); Alain Hugon, Au service du Roi Catholique. Honorables ambassadeurs et divins espions (Madrid: Casa de Vélazquez, 2004); Lucien Bély, Espions et ambassadeurs au temps de Louis XIV (Paris: Fayard, 1990).

${ }^{11}$ Sull'uso della categoria storiografica di resilienza nelle scienze sociali, cfr. Nicolas Marquis, "La résilience comme attitude face au malheur", SociologieS, Théories et recherches (2018), http://journals.openedition.org/sociologies/6633 (consultato il 3/02/2021). Un workshop dal titolo Femminili resilienze: storie, scritture e percorsi di ricerca tra XVI e XX secolo è stato organizzato a novembre 2020 dal Dipartimento di Storia Moderna e Contemporanea dell'Università Cattolica del Sacro Cuore a Milano.

${ }^{12}$ Cfr. Benedetta Craveri, L'áge de la conversation (Paris: Gallimard, 2002); Mathilde Bombart e Éric Méchouan, eds., Politiques de l'épistolaire au XVII siècle. Autour du Recueil Faret (Paris: Garnier, 2019); Giora Sternberg, "Epistolary Ceremonial: Corresponding Status at the Time of Louis XIV", Past and Present 204 (2009): 33-88. 
matrimonio fu oggetto di violenti conflitti e di un serrato dibattito giuridico ${ }^{13}$. La duchessa d'Orléans, costretta a separarsi dal marito, rimase confinata a Bruxelles fino al 1643, quando il re di Francia, ormai morente, le permise di raggiungere Gastone a Parigi. Nonostante una seconda benedizione nuziale, impartita a Meudon il 26 maggio dello stesso anno, Margherita visse sempre ai margini della famiglia reale, considerata, di fatto, come una parente indesiderabile dal nuovo re, dalla reggente Anna d'Austria e soprattutto dalla figliastra, Anna-Maria Luisa d'Orléans, figlia di primo letto di Gastone $^{14}$. La precarietà della sua situazione si acuì dopo il coinvolgimento del marito nei sollevamenti della Fronda, che costrinse il duca d'Orléans a ritirarsi a Blois, in una sorta di esilio volontario. Peraltro, le vicissitudini di Carlo IV, per lunghi anni prigioniero della Spagna, e le relazioni conflittuali tra la Francia e la Lorena resero ancora più delicata la posizione di Margherita alla corte di Luigi XIV ${ }^{15}$. L'insofferenza verso la propria condizione è evidente nella lettera che la principessa indirizza a Carlo Emanuele II, dopo che Luigi XIII ha finalmente acconsentito ad accoglierla a Parigi:

Après avoir éprouvé toutes les injustices que les ministres de France se pouvoient imaginer pour prendre leurs avantages dans la rupture de mon mariage et la désunion de la maison du Roi Monseigneur, la bonté divine a fait paroistre la vanité de leurs desseins et rendu la justice à la sincérité de mes actions [...] Leur décadence a donc ouvert les yeux de Sa Majesté dans la recognoissance des artifices dont ils se sont servis pour arrester le cours de ses bonnes et royales inclinations. Il me promet maintenant autant de part en ses bonnes grâces qu'ils se sont efforcés de m'en éloigner ${ }^{16}$.

Si badi che la debole posizione della duchessa d'Orléans, la cui legittimità è teoricamente incontestabile ma di fatto controversa, è esplicitamente riconosciuta sul piano delle relazioni diplomatiche con la corte sabauda. Quando gli ambasciatori di Vittorio Amedeo II dovranno negoziare il matrimonio di quest'ultimo con Anna Maria d'Orléans, secondogenita di Filippo d'Orléans, fratello di Luigi XIV, Vittorio Amedeo raccomanderà loro di non regolarsi sull'esempio di quanto si è seguito al momento del primo matrimonio di Carlo Emanuele. Non soltanto, egli scrive, Anna Maria è sorella della regina di Spagna e nipote di Luigi XIV - mentre, all'epoca delle nozze, Francesca

\footnotetext{
${ }^{13}$ Su Margherita di Lorena (1615-1672) mancano studi specifici: un'eccezione è il vecchio ma utile articolo di Lucien de Warren, "Marguerite de Lorraine, duchesse d'Orléans", Bulletin de la Société Philomatique Vosgienne (1882-1883): 137-175. Sulla maison della duchessa, cfr. Jonathan Spangler, "Bridging the Gaps: The Household Account Books of Marguerite de Lorraine, Duchesse d'Orléans", Annales de l'Est 2 (2017): 69-86. Su Gastone d'Orléans (1608-1660), cfr. Jean-Marie Constant, Gaston d'Orléans, prince de la liberté (Paris: Perrin, 2013); Pierre Gatulle, Gaston d'Orléans, entre mécénat et impatience du pouvoir (Seyssel: Champ Vallon, 2012).

${ }^{14}$ Secondo Anna-Maria Luisa d'Orléans, Anna d'Austria giudica molto severamente Margherita di Lorena: «Sa personne, son humeur et ses manières me sont odieuses» (Mémoires de Mademoiselle de Montpensier, ed. Adolphe Chéruel, Paris, Charpentier, 1858-1859, vol. 3, 371). Quanto ai rapporti fra Anna-Maria Luisa e Margherita, il celebre memorialista Saint-Simon non ha dubbi: «Elles se haissaient parfaitement» (Mémoires, ed. Arthur de Boislisle, Paris, Hachette, 1879-1931, t. 24: 29).

${ }_{15}$ Cfr. Anne Motta, Noblesse et pouvoir princier dans la Lorraine ducale, 1624-1737 (Paris: Garnier, 2016).

16 AsTo, Lettere Principi forestieri, m. 39: Margherita a Carlo Emanuele, 10 maggio 1643.
} 
Maddalena era solo la cugina del sovrano ${ }^{17}$; se non è concepibile, precisa Vittorio Amedeo, mettere sullo stesso piano Filippo d'Orléans e Margherita di Lorena, la stessa Elisabetta Carlotta di Baviera, seconda moglie di Filippo, non può essere trattata alla stregua della moglie di Gastone, «per la troppa differenza di condizione» ${ }^{18}$.

1.

Cristina di Francia, vedova di Vittorio Amedeo I e reggente per il figlio a partire dal 1638, ha saldamente mantenuto il ducato sabaudo nell'orbita francese, nonostante la guerra che l'ha opposta ai cognati, il principe Tommaso di Savoia-Carignano e il cardinale Maurizio ${ }^{19}$. Dopo la liberazione della Cittadella di Torino da parte delle truppe francesi, che l'avevano occupata durante la guerra civile, nel febbraio 1657, Cristina accelera $\mathrm{i}$ negoziati in vista di una doppia alleanza matrimoniale con la Francia $^{20}$.

Nell'autunno 1658, al termine di una lunga trattativa con il cardinale Mazzarino, Madama Reale si prepara a incontrare a Lione il nipote Luigi XIV, per proporgli in moglie sua figlia, Margherita Violante di Savoia ${ }^{21}$. Nel contempo, la duchessa, incoraggiata dal cardinale, intende negoziare l'unione fra Carlo Emanuele e Mademoiselle d'Orléans, Margherita Luisa, figlia primogenita di Gastone d'Orléans e di Margherita di Lorena. Il conte Alessandro Gerardo Scaglia di Verrua, colonnello della cavalleria del duca di Savoia, è quindi inviato al castello di Blois, dove vive Gastone d'Orléans, per osservare la principessa, «en ce qui regarde sa hauteur, sa taille, son port, sa façon et ses autres qualités tant du corps que de l'esprit ${ }^{22} \gg$.

\footnotetext{
${ }^{17}$ La prima figlia di Filippo d'Orléans, Maria Luisa, ha sposato nel 1679 Carlo II di Spagna.

18 AsTo, Matrimoni, m. 36: istruzione di Vittorio Amedeo II al conte Ferrero, 1684.

${ }_{19}^{19}$ Giuliano Ferretti, dir., Les États de Savoie, du duché à l'Unité d'Italie (1416-1861) (Paris: Garnier, 2020), 261-283; Lucien Bély, “Le Piémont-Savoie au coeur des conflits européens”, in Ferretti, L'État, la cour et la ville, 93-121; Blum, La diplomatie de la France, 97-135.

${ }^{20}$ Cfr. Daniel Grange, "Le duché de Savoie face à l'hégémonie française au XVII" siècle: un État sous haute surveillance (1631-1690)", in La Savoie et ses voisins dans l'histoire de l'Europe (Annecy: Académies Florimontane et Salésienne, 2010), 129-142; Lucien Bély, "Christine de France, duchesse de Savoie, et la politique française au temps de Mazarin", e Pierpaolo Merlin, "Vassal de la France ou État de Souverain? Le duché de Savoie et les traités de Westphalie (1641-1648)", Revue Dix-Septième Siècle 262 (2014): 21-29, 31-42. Qualche anno prima, Cristina di Francia aveva progettato un matrimonio fra Carlo Emanuele e Caterina di Braganza, infanta del Portogallo: AsTo, Matrimoni, m. 30, fasc. 1, Istruzione di Madama Reale a un padre francescano inviato segretamente a Lisbona, dicembre 1653. Si era anche parlato di Enrichetta Anna Stuart, sorella di Carlo II d'Inghilterra, ma il duca si era opposto a un'alleanza con il sovrano, allora in esilio: Lettere Ministri, Francia, m. 72, Ghiron Villa a Carlo Emanuele, 15 aprile 1661.

${ }^{21}$ Sul fallito progetto di matrimonio fra Luigi XIV e Margherita Violante, cfr. Michel Bougain, "La comédie de Lyon: relecture d'un mythe historique", Revue Dixseptième Siècle 274 (2017): 129-59; Daniel Serré, "Mazarin et la Comédie de Lyon: au-delà de la légende", Ibidem 231 (2006): 327-340.

22 Matrimoni, m. 30, fasc. 3: istruzione di Madama Reale al conte di Verrua, 13 febbraio 1658. Nelle sue memorie, Anna-Maria Luisa evoca rapidamente la visita del conte: Mémoires de Mademoiselle, vol. 3, 216, 229-31.
} 
L'idea di un matrimonio fra Margherita Luisa e Carlo Emanuele si inserisce nel contesto della tradizionale rivalità fra la dinastia sabauda e quella dei Medici, che contendono ai duchi di Savoia la precedenza cerimoniale nelle corti europee: ad aspirare alla mano di Mademoiselle d'Orléans è infatti anche Cosimo de' Medici, erede del granduca Ferdinando $\mathrm{II}^{23}$. In realtà, però, Cristina di Francia aveva pensato in prima istanza ad Anna-Maria Luisa d'Orléans, la celebre Grande Mademoiselle, figlia di Gastone e della sua prima moglie Maria di Borbone, duchessa di Montpensier. AnnaMaria Luisa, che è stata l'anima della Fronda contro Mazzarino, è la più ricca ereditiera di Francia, grazie all'immensa fortuna legatale da sua madre ${ }^{24}$. Ma i rapporti fra AnnaMaria Luisa e Luigi XIV sono difficili; inoltre, il temperamento orgoglioso e collerico della principessa non la rende certo una nuora ideale per Cristina, poco incline a fare spazio a una personalità ingombrante, che non tarderebbe a creare tensioni. Del resto, l'incontro fra Carlo Emanuele e Anna-Maria d'Orléans a Lione è un fallimento: i due principi non sembrano manifestare il minimo interesse reciproco ${ }^{25}$. In compenso, il duca di Savoia si mostra attratto da Maria-Giovanna Battista di Savoia-Nemours, figlia di Isabella di Bourbon-Vendôme, vedova di Carlo Amedeo di Savoia-Nemours, duca del Genevois ${ }^{26}$. Un fidanzamento del duca di Savoia con Maria-Giovanna Battista è tuttavia scartato, per ragioni diverse, sia da Madama Reale che da Mazzarino. Se quest'ultimo, infatti, auspica per la principessa un matrimonio francese, che consentirebbe a Luigi XIV di mantenere un maggiore controllo sul suo immenso patrimonio, distribuito tra la Francia e la Savoia, Cristina teme probabilmente che Maria-Giovanna Battista acquisisca un'eccessiva influenza sul figlio, trasformandosi in una scomoda rivale ${ }^{27}$.

${ }^{23}$ Cfr. Toby Osborne, "The surrogate war between the Savoys and the Medici: Sovereignty and Precedence in Early Modern Italy", The International History Review 29 (2007): 1-21; Franco Angiolini, "Medici e Savoia: contese per la precedenza e rivalità di rango in età moderna", in L'affermarsi della corte sabauda. Dinastie, poteri, élites in Piemonte e Savoia fra tardo medioevo e prima età moderna, eds. Paola Bianchi e Andrea Merlotti (Torino: Zamorani, 2006), 435-80.

${ }^{24}$ Su Anna-Maria Luisa d'Orléans (1627-1693), cfr. Vincent Pitts, La Grande Mademoiselle at the court of France: 1627-1693 (London: The Johns Hopkins University Press, 2000). Sulla sua partecipazione alla Fronda, cfr. Sophie Vergnes, Les Frondeuses. Une révolte au féminin (1643-1661) (Seyssel: Champ Vallon, 2013); sul suo patrimonio, cfr. Bernard Allorent, La fortune de la Grande Mademoiselle (Paris: Champion, 2019).

${ }^{25}$ L'incontro con Carlo Emanuele è raccontato dalla Grande Mademoiselle: Mémoires, vol. 3, 313315.

${ }^{26}$ Sulla famiglia dei Savoia-Nemours, cfr. Mathew Vester, Jacques de Savoie-Nemours. L'apanage du Genevois au cæur de la puissance dynastique savoyarde au XVI siècle (Genève: Droz, 2008).

${ }^{27}$ Cfr. Géraud Poumarède, "Mazarin marieur de l'Europe. Stratégies familiales, enjeux dynastiques et géopolitique au milieu du XVIIe siècle", Revue Dix-Septième Siècle 243 (2009): 201-218. Su MariaGiovanna Battista di Savoia Nemours, che sposerà Carlo Emanuele nel 1665, cfr. Blythe A. Raviola e Clelia Arnaldi, eds., Maria-Giovanna Battista di Savoia-Nemours (Torino: Allemandi, 2017); Robert Oresko, "Princesses in Power and European Dinasticism: Marie-Christine of France and Navarre and Maria Giovanna Battista of Savoy-Genevois-Nemours", in In assenza del Re. Le Reggenti nei secoli XVT-XVII (Piemonte ed Europa), ed. Franca Varallo (Firenze: Olschki, 2008), 393-434; Idem, "Maria-Giovanna Battista of Savoy-Nemours (1664-1724): daughter, consort and regent of Savoy", in Queenship in Europe 1660-1815, dir. Clarissa Campbell-Orr (Cambridge: CUP, 2004), 16-55. 
Dopo la morte di Gastone d'Orléans, nel febbraio 1660, e la conclusione del matrimonio fra Luigi XIV e l'infanta Maria Teresa, Ferdinando II de' Medici accelera le negoziazioni per le nozze fra Cosimo e Mademoiselle d'Orléans; grazie all'appoggio determinante della curia romana, il contratto di nozze è firmato nell'aprile $1661^{28}$. L'accordo rappresenta senza dubbio un insuccesso diplomatico per Cristina e per il duca di Savoia, uscito sconfitto anche dalla competizione per la mano di Eleonora di Gonzaga-Nevers, vedova dell'imperatore Ferdinando III ${ }^{29}$. Madama Reale si volge dunque verso la terzogenita di Gastone e Margherita, la quattordicenne Mademoiselle di Valois.

Sia Gastone che Margherita avevano rifiutato di partecipare al fallimentare incontro di Lione, giustificandosi con un'indisposizione che impediva loro di viaggiare: ma è probabile che la duchessa volesse soprattutto evitare le prevedibili controversie di precedenza con Cristina ${ }^{30}$. Secondo Anna-Maria Luisa d'Orléans, Madama Reale non aveva apprezzato l'assenza di Margherita, giudicandola «fort ridicule» ${ }^{31}$. In ogni caso, nel giugno 1661, Cristina ordina al marchese Ghiron Villa di recarsi al palazzo del Luxembourg, dove vive la duchessa vedova: il re le ha infatti impedito di ritirarsi presso il monastero parigino delle benedettine del Santo Sacramento, fondato dalla nobildonna lorenese Caterina di $\mathrm{Bar}^{32}$. Ghiron Villa può osservare le due principesse rimaste nubili, Mademoiselle di Valois e Mademoiselle d'Alençon, Elisabetta Margherita d'Orléans, per la quale si prospetta un'unione con il principe ereditario di Danimarca ${ }^{33}$. Egli rileva che Mademoiselle di Valois «ha volto assai bello e la pelle di bellissimo colore, e non vi è nessuna marca né rossore, e mi pare abbia buona grazia nel parlare ${ }^{34}$. L'estate seguente, Margherita permette a un paggio della marchesa Camilla Bevilacqua, moglie di Ghiron Villa, di introdursi nella camera delle due principesse per osservarle mentre si truccano ${ }^{35}$. I rapporti inviati a Madama Reale e a Carlo Emanuele sono decisamente favorevoli a Mademoiselle di Valois, sebbene la marchesa la trovi «alquanto languidetta» e di salute cagionevole ${ }^{36}$.

2.

Ancor prima che si aprano ufficialmente le trattative, Margherita di Lorena e gli inviati sabaudi si scontrano con l'ostilità della Grande Mademoiselle. Quest'ultima, infatti, si oppone all'idea che le figlie di secondo letto di Gastone trovino marito prima

\footnotetext{
${ }^{28}$ Su Margherita Luisa d’Orléans (1645-1721), cfr. Vincenzo Lagioia, La verità delle cose. Margherita Luisa d'Orléans: donna e sovrana d'ancien régime (Roma: Edizioni di Storia e Letteratura, 2015).

${ }^{29}$ Matrimoni, m. 30, fasc. 4: Istruzione del duca Carlo Emanuele II a Gio. Pietro Quaglino, maggio 1660.

${ }^{30}$ Lettere Principi forestieri, m. 39: Margherita a Madama Reale, 29 novembre 1658.

31 Mémoires de Mademoiselle, vol. 3, 317.

32 Cfr. Daniel O. Hurel, "L'itinéraire de Catherine de Bar (1614-1698) et l'oeuvre de René Taveneaux", in Dorsale catholique, Jansénisme, Dévotions: XVIe-XVIII e siècles, Mythe, réalité, actualité bistoriographique (Paris: Riveneuve, 2014), 291-302.

33 Elisabetta Margherita d'Orléans (1646-1696) sposerà Luigi Giuseppe di Lorena, duca di Guisa.

${ }^{34}$ Lettere Ministri, Francia, m. 72: Ghiron Villa a Carlo Emanuele, 18 giugno 1661.

35 Ibidem, m. 73: la marchesa Villa a Madama Reale, 24 giugno 1662.

${ }^{36}$ Ibidem, 10 ottobre 1662.
} 
di lei ${ }^{37}$. Ghiron Villa vi accenna in una lettera a Madama Reale dell'agosto 1661, ma è soprattutto Margherita a inquietarsi degli intrighi della figliastra. Anna-Maria Luisa, spiega Margherita alla marchesa Bevilacqua, è stata immediatamente informata delle trattative confidenziali per il matrimonio di Mademoiselle di Valois: ella ha inviato a Luigi XIV una lettera piena di recriminazioni, «in cui rendeva grazie al Re della cura che si prendeva di maritare le sue sorelle cadette, e particolarmente Mademoiselle di Valois, sperando che Sua Maestà avrebbe poi anche la bontà di applicarsi per provvedere a lei» ${ }^{38}$.

Per evitare che la cugina crei scompiglio a corte, il re le impone di ritirarsi, fino a nuovo ordine, nelle sue terre di Saint-Fargeau. Tuttavia, malgrado la semi-disgrazia, la Grande Mademoiselle può contare su numerosi alleati. Nelle lettere di Margherita di Lorena e in quelle degli agenti di Madama Reale vediamo effettivamente delinearsi una «cabala», che osteggia in tutti i modi il matrimonio sabaudo. Al centro vi è Anna-Maria Luisa, che Margherita designa con una perifrasi: «la personne qu'on a éloignée de la cour ${ }^{39} \gg$. Vi fanno parte, in primo luogo, Enrichetta Anna Stuart, moglie di Filippo d'Orléans, il principe Luigi di Borbone-Condé e suo figlio, il duca d'Enghien, il quale, secondo Margherita, aspira alla mano di Mademoiselle di Valois. Filippo d'Orléans è anch'egli individuato come elemento ostile, sebbene in subordine alla moglie ${ }^{40}$. Margherita colloca fra i propri nemici anche Mademoiselle de La Vallière, la giovane favorita di Luigi XIV, sobillata dal patrigno, Jacques de Courtavel marchese di Saint Remi, primo maître d'bôtel di Gastone: Margherita ha infatti provocato l'ira del marchese, ostacolando la passione del duca di Lorena per sua figlia, Caterina de Courtavel ${ }^{41}$.

La condotta della Grande Mademoiselle appare in effetti estremamente ambigua. Da un lato, la principessa rifiuta ostinatamente tutti i partiti che le vengono proposti, in ultimo il re del Portogallo, Alfonso VI; dall'altro, ella insiste presso il re affinché le trovi un marito adatto, lamentandosi che il sovrano abbia troppo a cuore la sorte delle sorellastre. Anna-Maria Luisa riempie le sue lettere a Madama Reale di ardenti protestazioni di amicizia. Al fine di controbattere a chi, come Anna d'Austria, la accusa di non mostrare abbastanza rispetto per Cristina ${ }^{42}$, la principessa si appella a Madame de Choisy, vedova del cancelliere di Gastone d'Orléans e madre del celebre memorialista. La chancelière d'Orléans, come si firma nelle sue lettere a Cristina, è una

${ }^{37}$ Nel 1659, Madama Reale aveva accusato la Grande Mademoiselle di spargere calunnie sul fisico infelice di Mademoiselle d'Orléans. Anna-Maria Luisa aveva contestato vigorosamente le accuse, scrivendo una lettera «la plus fière que l'on se puisse imaginer» a Cristina e inviando a Torino un suo gentiluomo. Mémoires de Mademoiselle, vol. 3, 392-395.

${ }^{38}$ Lettere Ministri, Francia, m. 73: Ghiron Villa a Carlo Emanuele, 20 ottobre 1662.

${ }^{39}$ Lettere Principi forestieri, m. 39: Margherita a Madama Reale, 11 gennaio 1663. Il termine «cabala», per designare gli alleati di Mademoiselle di Montpensier, è utilizzato sia da Margherita sia da Ghiron Villa.

${ }^{40}$ Lettere Ministri, Francia, m. 73: Ghiron Villa a Carlo Emanuele, 8 novembre 1662.

${ }^{41}$ Ibidem.

${ }^{42}$ Sui frequenti rimproveri di Anna d'Austria per lo scarso rispetto dimostrato verso Cristina e la condotta ambigua nei confronti di Carlo Emanuele, si veda la corrispondenza della Grande Mademoiselle con Madama Reale: Lettere principi forestieri, m. 30, in particolare le lettere del 3, 17 e 24 settembre 1660 . 
confidente di Margherita e svolge una vera e propria attività di spionaggio per Madama Reale, grazie a una rete di informatori distribuiti fra Parigi, Lisbona e Nancy ${ }^{43}$. AnnaMaria Luisa la prega di intervenire in suo favore presso Cristina ${ }^{44}$; nel contempo, tuttavia, la principessa avverte Luisa Cristina di Savoia, figlia primogenita di Cristina, affinché metta in guardia Madama Reale dagli intrighi di Madame de Choisy ${ }^{45}$. A sua volta, quest'ultima denuncia a Cristina la malafede della Grande Mademoiselle, che rimprovera il re di non curarsi dei suoi interessi matrimoniali, ma nello stesso tempo crea ogni sorta di difficoltà ai possibili pretendenti, cercando inoltre di impedire alle sorellastre di convolare a nozze ${ }^{46}$.

Margherita di Lorena dispone di un'altra alleata per convincere Carlo Emanuele a sposare Mademoiselle di Valois: si tratta della nobildonna bretone JeanneMarie di Trécesson, marchesa di Cavour, favorita del duca di Savoia, da cui ha già avuto due figli. Jeanne-Marie è giunta alla corte di Torino nel luglio 1658, come figlia d'onore di Madama Reale, grazie alla protezione del sovrintendente delle Finanze di Luigi XIV, il celebre Nicolas Fouquet, e del conte Anne de Brûlon, già introducteur des ambassadeurs del re di Francia; ella è, inoltre, nipote di Suzanne de Bruc du Plessis-Bellière, grande

${ }^{43}$ AsTo, Lettere di particolari, C, m. 80, Choisy de Caen, 1659-1663. Sull'amicizia fra Madame de Choisy e Margherita di Lorena, cfr. Lettere Ministri, Francia, m. 73, Ghiron Villa a Carlo Emanuele, $1^{\circ}$ dicembre 1662.

${ }^{44}$ Lettere Ministri, Francia, m. 73: il senatore Pietro Carroccio a Carlo Emanuele, 10 ottobre 1662.

${ }^{45}$ Lettere Principi forestieri, m. 30, la Grande Mademoiselle a Luisa Cristina di Savoia, 17 settembre 1660: «'ai appris que M.me de Choisy, femme du chancelier de Monsieur mon père, écrit tous les ordinaires à S.A.R. et qu'elle s'est vantée d'en avoir des lettres, par lesquelles S.A.R. lui parle de moi dans les termes le plus désobligeantes du monde, sans me nommer. J'ai écrit à Madame Royale; je vous conjure, au nom de Dieu, de découvrir ce qu'il en est et d'en parler à Madame Royale, et même si M. votre frère voudrait en parler à Madame Royale et que vous deux ensemble voudraient prendre mes intérêts auprès d'elle pour effacer cette impertinence, je vous en aurais beaucoup d'obligation, rien au monde ne m'étant plus cher que l'honneur de ses grâces. [...] Elle est très mal auprès de la Reine mère, ayant été chassée pendant la maladie du Roi pour des critiques qu'elle fit contre la Reine mère et M. le Cardinal [...] Enfin, c'est une intrigante, une brouillonne qui n'a jamais fait que du mal à tout le monde et qui, ne se contentant pas d'en faire en France, veut en procurer et à Madame Royale et à M. votre frère, et par conséquence à tout le Piémont».

${ }^{46}$ Lettere di particolari, C, m 80, Choisy de Caen, s. d. [1662]: «Mademoiselle envoya son premier écuyer à la Reine mère, pour la supplier de savoir du Roi s'il lui serait agréable qu'elle s'épousât l'Archiduc [Leopoldo Guglielmo d'Austria, fratello dell'imperatore Ferdinando III]. Le Roi dit qu'il lui serait très agréable. Ensuite, cet ambassadeur n'étant pas du calibre dont on les fait d'ordinaire, l'on a écrit à Mademoiselle que les courtisans avoient un peu grondé son choix: ce qui l'a fait résoudre d'écrire ici qu'il avoit parlé sans procuration et qu'elle n'en vouloit point, si le Roi d'Espagne ne lui donnoit pas les Pays Bas à gouverner. Vous jugerez ce que le Roi d'Espagne en dira». 
amica di Fouquet ${ }^{47}$. Cristina l'ha presa sotto la propria ala, incoraggiando discretamente la sua relazione con Carlo Emanuele ${ }^{48}$.

Durante i negoziati per il matrimonio fra Luigi XIV e Margherita Violante, Madama Reale si è servita di Mademoiselle di Trécesson per tenersi segretamente in contatto con Fouquet, fervente sostenitore del matrimonio sabaudo contro Mazzarino, favorevole invece all'unione con l'infanta Maria Teresa ${ }^{49}$. Jeanne-Marie ambiva a diventare figlia d'onore di Margherita Violante, una volta che quest'ultima avesse cinto la corona di Francia ${ }^{50}$. Dopo il fallimento delle trattative, la giovane è rimasta a Torino, dove Cristina ha combinato il suo matrimonio con Maurizio-Pompilio Benso, marchese di Cavour ${ }^{51}$. Madama Reale ha continuato a incoraggiare la relazione fra suo figlio e Jeanne Marie, della quale si serve per comunicare confidenzialmente con Fouquet ${ }^{52}$. Commentando con quest'ultimo i progetti riguardanti Mademoiselle di Valois e il duca di Savoia, Jeanne-Marie rileva che, non appena Anna-Maria Luisa

47 AsTo, Lettere di Particolari, B, m. 125: il conte di Brûlon a Madama Reale, 21 giugno 1658. JeanneMarie (1634-1677) è figlia di Paul Carné, visconte di Trécesson, e di Jeanne de Bruc, sorella di Suzanne du Plessis-Bellière. La sola biografia di Jeanne-Marie è quella di Giorgio Peyron, Jeanne-Marie de Trécesson, marchesa di Cavour (Savigliano: L'Artistica Savigliano, 1990). Le informazioni che seguono si ricavano dalle lettere di Jeanne-Marie a Nicolas Fouquet, segnalate da A. Chéruel, Mémoires sur la vie publique et privée de Fouquet (Paris: Charpentier, 1862), vol. 2, 485-486. Le lettere, recuperate nella cassetta di Fouquet al momento del suo arresto, si trovano a Parigi, Bibliothèque Nationale [BNF], Manuscrits, Baluze 149, vol. 1, f. 54; 150, vol. 2, f. 202-204, 211-213, 245-249, 254-256, 280-284, 313-319, 332-333. Tutte le persone menzionate sono indicate con pseudonimi; Jeanne-Marie parla di se stessa e di Fouquet alla terza persona, usando gli pseudonimi di Mademoiselle de Bellairt e Monsieur le Baron; le lettere portano spesso la data topica fittizia de Caen. Un'attenta lettura consente di identificare con sufficiente sicurezza quasi tutti i personaggi citati.

${ }^{48}$ BNF, Baluze 150, vol. 2, f. 249v.-250r., Mademoiselle di Trécesson a Fouquet, 25 ottobre 1658: «Je vous dirai qu'il ne se passe rien entre Mlle de Bellairt [Jeanne-Marie] et M. du Clos [Carlo Emanuele] qui soit désavantageux ni pour vous ni pour elle: elle a trouvé le moyen de s'en faire craindre et de s'en faire estimer malgré lui. Elle a toujours pris en raillant ce qu'il lui a dit de plus sérieux, il lui parle tout autant qu'il le peut, par l'ordre de M.me Aubert [Madama Reale], qui est bien aise que cette demoiselle ait quelque crédit auprès de lui [...] M.me Aubert lui a donné depuis peu des boucles de diamants; j’ai su depuis huit jours que les perles dont elle lui avoit fait un présent venoient de M. du Clos, qui avoit obligé cette dame à les lui donner comme venant d'elle». Lettera pubblicata in A. Chéruel, Mémoires, vol. 1, 502-505, citata da Ricotti, Storia della monarchia, vol. VI, 156-158.

${ }^{49}$ Baluze 150, vol. 2, f. 257v., 2 novembre [1658]: «Il faut que je revienne à Mlle le Roy [Margherita Violante] et que je vous fasse encore quelque réponse sur son chapitre. C'est que je ne la crois pas assez hardie pour oser résister en rien à $\mathrm{M}$. le Conseiller [Mazzarino], mais elle aimera toujours ceux à qui elle aura promis amitié et ne manquera jamais de reconnaissance pour les personnes qui l'auront obligée».

50 Ibidem, f. 253r., 25 ottobre 1658: «Si le mariage que vous savez s'accorde, je vous supplierai de prendre la peine d'écrire à Mme Aubert, afin qu'elle donne Mlle de Bellairt à Mlle le Roy».

${ }^{51}$ Ibidem, f. 333r, 20 mai 1659: «Je sais que la personne à qui Mme Aubert a pensé pour Mlle de Bellairt est de la plus grande qualité et aura un jour plus de cent mille livres de rente».

52 Ibidem, f. 254, 28 febbraio [1660]: «Mme Aubert vous assure qu'elle prend beaucoup de part à la nouvelle preuve que vous avez reçue, de l'estime de M. le Président [Luigi XIV] et de M. le Conseiller, et je vous assure qu'elle témoigne pour M. Le Baron plus de reconnaissance qu'on ne sauroit imaginer»; Lettere di Particolari, B, m. 125: il conte di Brûlon a Cristina di Francia, 5 settembre 1659. 
d'Orléans ne ha avuto sentore, essa è ricorsa ad Anna d'Austria, per convincere Madama Reale a riconsiderare, piuttosto, la sua candidatura ${ }^{53}$.

Gli inviati sabaudi sono concordi con Margherita di Lorena, Madame de Choisy e Mademoiselle di Trécesson nel denunciare la malafede di Anna-Maria Luisa. Ghiron Villa osserva malignamente che, in realtà, il vero cruccio della Grande Mademoiselle è quello «di non possedere quella fresca e giovanile età e quella vaghezza di cui Mademoiselle di Valois si trova tanto ben provveduta» ${ }^{54}$.

3.

Nel novembre 1662, il marchese Vittorio Pallavicino delle Frabose, gentiluomo di camera di Carlo Emanuele II, arriva a Parigi per chiedere la mano di Mademoiselle di Valois in nome del duca. Margherita di Lorena si mostra impaziente di concludere il matrimonio e fa pressione su Luigi XIV affinché accolga favorevolmente la proposta di Carlo Emanuele: «Je vous supplie de croire», scrive la principessa a Madama Reale, «que je n'ai rien omis pour vous rendre en cette rencontre tous les services auprès du Roi que se pouvoit, et je dois vous dire que S.M. m'a parlé si obligeamment que je souhaitais que vous pouissiez l'un et l'autre l'entendre, car je suis persuadée que vous en auriez été satisfaits ${ }^{55} \%$.

Allorché il marchese Pallavicino è autorizzato a rendere pubblico il fidanzamento, Margherita informa immediatamente Cristina:

Je ne peux vous exprimer la joie que j'ai de voir enfin comme Dieu bénit nos désirs sous votre conduite très prudente. Je m'assure que M. votre ambassadeur vous mande exactement tout le détail de ce qui est arrivé depuis qu'il a reçu l'ordre de S.A.R. mon neveu de faire la demande au Roi de ma fille. C'est ce matin que le Roi lui a donné pour ce sujet une audience secrète de laquelle il a sujet d'être content par l'accueil que S.M. lui a fait et par le consentement qu'il a donné ${ }^{56}$.

Nelle sue memorie, la Grande Mademoiselle è laconica al riguardo: «Ma bellemère fit si bien par ses intrigues en Savoie, que Madame Royale se résolut de marier son fils avec ma soeur de Valois ${ }^{57}$. Certamente Margherita vede nella conclusione dell'accordo un successo personale, ma soprattutto un debito contratto con Cristina. La vedova di Gastone si considera, ormai, come una protégée di Madama Reale:

${ }^{53}$ Baluze 150, vol. 2, f. 204v, 22 marzo [1661]: «L'on a mandé à Mme Aubert ce dernier ordinaire que Mademoiselle a prié Mme la Présidente [Anna d'Austria] de la proposer à Madame Royale de Savoie pour sa belle-fille, et que l'on lui a répondu qu'il y avait déjà des propositions pour Mademoiselle de Valois sa sœur, qui seroient assurément approuvées de part et d'autre [...] Mme Aubert craint fort cette personne, et soupçonne qu'elle n'ait intelligence avec M. Du Clos». La Grande Mademoiselle è al corrente dell'amicizia tra Fouquet e Jeanne-Marie, ma ignora che Madama Reale si serve della Trécesson per comunicare con il sovrintendente: Mémoires, vol. 3, 311.

54 Matrimoni, m. 30, fasc. 5: Ghiron Villa a Carlo Emanuele, 14 gennaio 1663.

${ }^{55}$ Lettere Principi forestieri, m. 39: $1^{\circ}$ gennaio 1663.

${ }^{56}$ Ibidem, s. d. [dicembre 1662].

57 Mémoires de Mademoiselle, vol. 3, 566. 
Je ne sais comme quoi je pourrai vous faire cognoistre l'excès de joie, qui est telle qu'elle ne se peut comprendre. Vous le jugerez, s'il vous plait, par le désir de tant d'années à voir les choses au point où je le vois présentement, que si je pouvois mourir de joie cela auroit été en recevant votre lettre, qui m’apprend la résolution de S.A.R. mon neveu à l'égard du mariage de ma fille de Valois. Enfin, Madame et très chère sœur, je n'auroi plus autres soins dorénavant que de pouvoir vous faire voir, par toutes mes actions et par celles de ma fille, que nous ne souhaitons plus rien au monde que de vous donner par effet des marques de nos soumissions et d'une éternelle recognoissance ${ }^{58}$.

Il duca di Savoia può ormai cominciare il tradizionale corteggiamento epistolare, inviando alla fidanzata ricchi doni, accompagnati da lettere galanti e brevi componimenti poetici ${ }^{59}$. Sfortunatamente, gli amici di Anna-Maria Luisa diffondono a corte il loro contenuto, prendendosi gioco delle velleità letterarie di Carlo Emanuele ${ }^{60}$. L'offensiva scatenata dalla Grande Mademoiselle accresce il senso di isolamento di Margherita, convinta di essere circondata da nemici. «Il faut bien, ma chère sœur», ella scrive a Madama Reale, «que vous n'ayez pas assez averti S.A.R. de toutes les cabales qui se devoient former par les esprits envieux de son bonheur et du nôtre, aussitôt que son mariage seroit declaré» ${ }^{61}$. Nonostante l'appoggio del sovrano, «qui m'a fait dire que je ne m'en mette point en peine, et que c'est assez que S.A.R. mon neveu ait choisie celle de ses cousines qu'il aime mieux, qu'il s'en soit déclaré et qu'il en ait fait la demande, qu'après cela il ne croit pas qu'il y ait d'intrigues ni d'entreprises qui puissent empêcher ou troubler cette affaire», Margherita teme «les mille malicieuses traverses des esprits mal faits et malins que vous connaissez». Ella confessa a Madama Reale di attendere con ansia il giorno della partenza della figlia, «car je vous avoue que je crains à présent mille choses que vous pouvez imaginer, jusqu'à ce qu'elle soit entre vos mains et hors de la vue de ceux qui ne pourront souffrir son bonheur» ${ }^{62}$.

Margherita di Lorena è attanagliata dall'angoscia, tanto da temere che gli alleati della figliastra siano «assez galliards pour faire quelque folie» il giorno stesso delle nozze. Per questa ragione, ella indirizza a Madama Reale una richiesta sorprendente. Conformemente alla tradizione, il matrimonio deve svolgersi per procura, nella patria della sposa: una volta che questa sarà giunta in territorio sabaudo, si procederà ad un'ulteriore benedizione nuziale in presenza del marito. Carlo Emanuele ha inviato una procura a Eugenio Maurizio di Savoia-Carignano, conte di Soissons, perché sposi Mademoiselle di Valois in suo nome: ma Margherita insiste perché egli invii una

\footnotetext{
${ }^{58}$ Lettere Principi forestieri, m. 39: 6 ottobre 1662.

${ }^{59}$ Un sonetto di Carlo Emanuele, dedicato a Mademoiselle di Valois, si trova nel Recueil Conrart, BNF, Bibliothèque de l'Arsenal, Ms-5422, t. XIII, 305.

${ }^{60}$ Matrimoni, m 30, fasc. 6: Ghiron Villa a Carlo Emanuele, 31 gennaio 1663. Nelle sue memorie, la Grande Mademoiselle ricopia uno dei biglietti del duca di Savoia, ironizzando sul suo stile: Mémoires, vol. 3, 569 .

${ }^{61}$ Lettere Principi forestieri, m. 39: 13 dicembre 1662.

${ }^{62}$ Ibidem, s.d. [dicembre 1662].
} 
seconda procura a Ghiron Villa, affinché quest'ultimo possa sposare la principessa la sera prima delle nozze, alla presenza di testimoni fidati. Ella spiega a Cristina:

J'écris encore ce mot pour vous prier de bien faire entendre, en particulier et secrètement, à S.A.R. qu'il ne peut assez s'imaginer comme lui et moi dans cette occasion avons sujet d'y faire considération, à cause de la furieuse colère dans laquelle est la personne que l'on a éloignée de la cour. Comme c'est de l'intérêt de S.A.R., je vous supplie bien fort de lui faire prendre mon avis sérieusement et de lui recommander le secret, afin que le monde ne tourne pas la chose en ridicule: et de le prier de trouver bon, outre le pouvoir qu'il a envoyé pour le comte de Soissons pour épouser, d'en envoyer encore très secrètement un autre au marquis Ville, afin que le marquis, la veille de la cérémonie, sous prétexte d'une visite chez moi, puisse, sans que l'on sache, en présence d'un prêtre et de deux témoins que je choisirai tel qu'il les faut, faire l'essentiel de la cérémonie, qui se répétera le lendemain devant tout le monde. Et l'on n'en saura rien, et sans fâcher personne. Mais le principal est que S.A.R. commande au marquis Ville de n'en parler à qui que ce soit, et n'en communiquer qu'avec moi ${ }^{63}$.

Né le lettere superstiti di Margherita né il carteggio degli inviati sabaudi consentono di stabilire se la cerimonia «clandestina» ha avuto luogo: ma Carlo Emanuele ha effettivamente inviato a Margherita una seconda procura per Ghiron Villa ${ }^{64}$.

La scelta di colui che dovrà ufficialmente rappresentare Carlo Emanuele II è un'ulteriore fonte di inquietudine per Margherita. Essa è legata alla vexata quaestio della dignità reale e della preminenza sugli altri principi italiani a cui aspirano i duchi di Savoia, in quanto principi dell'Impero e discendenti di Ottone III ${ }^{65}$. Cristina di Francia vorrebbe, infatti, che la futura duchessa di Savoia fosse portata all'altare da un principe del Sangue o meglio ancora dal fratello di Luigi XIV, Filippo d'Orléans. Dopo qualche tentennamento, quest'ultimo rifiuta, nell'intento, secondo Margherita, di compiacere sua moglie e soprattutto la Grande Mademoiselle, che gli avrebbe promesso in cambio di legare una parte dei propri beni ai suoi figli ${ }^{66}$.

Se il duca d'Orléans si oppone alle pretese di Carlo Emanuele, il conte di Soissons è, al contrario, ben lieto di offrirsi come rappresentante del cugino. Egli suscita però i sospetti di Margherita, che, d'altra parte, è in pessimi rapporti con Eugenio Maurizio e con sua moglie Olimpia Mancini. Nelle lettere di Margherita

63 Ibidem, 11 dicembre 1662.

${ }^{64}$ Matrimoni, m. 30, fasc. 5, 19 dicembre 1662.

${ }^{65}$ Cfr. Saniye Al-Baghdadi, "La dynastie de Savoie et le traitement royal au XVIIe siècle", in Ferretti, De Paris à Turin, 229-46; Géraud Poumarède, "Deux têtes pour une couronne: la rivalité entre la Savoie et Venise pour le titre royal de Chypre au temps de Christine de France", Revue Dix-septième siècle 262 (2014): 53-64; Robert Oresko, "The House of Savoy in search of a Royal Crown in the Seventeenth Century", in Royal and Republican Sovereignty in Early Modern Europe, ed. Graham C. Gibbs, Robert Oresko e H. M. Scott (Cambridge: CUP, 1997), 272-350. Sulle pretese origini sassoni della dinastia sabauda e sui suoi rapporti con l'Impero, cfr. Marco Bellabarba e Andrea Merlotti, ed., Stato Sabaudo e Sacro Romano Impero (Bologna: Il Mulino, 2014).

${ }^{66}$ Matrimoni, m. 30, fasc. 5: Ghiron Villa a Carlo Emanuele, 8 novembre 1662. 
vediamo dunque delinearsi un secondo gruppo ostile al matrimonio sabaudo, quello dei principi di Carignano-Soissons: vale a dire Olimpia Mancini, suo marito, la madre di quest'ultimo, Maria di Borbone, principessa di Carignano, e sua sorella Luisa Cristina di Savoia-Carignano. I Carignano-Soissons, principi del Sangue alla corte sabauda e princes étrangers alla corte di Francia, suscitano la più grande diffidenza, tanto a Parigi quanto a Torino, in quanto molto vicini alla Spagna e agli Asburgo d'Austria ${ }^{67}$. Tornato da una visita a Maria di Borbone, il marchese Pallavicino rileva che a palazzo Carignano «non si sentono che esclamazioni e contro Madama la duchessa d'Orléans e contro un'infinità di altri suoi parziali» ${ }^{68}$. Occorre ricordare che, a quest'epoca, Eugenio Maurizio di Soissons è l'erede presuntivo di Carlo Emanuele immediatamente dopo suo fratello, Emanuele Filiberto di Savoia-Carignano, che molti considerano inadatto a governare. Nell'estate 1662, il duca di Savoia cade seriamente ammalato, tanto da temere per la sua vita: alla corte di Torino c'è chi sospetta il conte di Soissons di essere pronto a rivendicare la successione, con l'appoggio di Luigi $\mathrm{XIV}^{69}$.

Il clima di sospetto che aleggia intorno ai Carignano-Soissons è palpabile nelle lettere di Margherita: «il est important de faire en sorte que le comte de Soissons ne soit pas choisi pour épouser ma fille», ella scrive a Madama Reale ${ }^{70}$. In realtà, ciò che la inquieta non è tanto il ruolo affidato al conte il giorno delle nozze, quanto il fatto che egli possa accompagnare Francesca Maddalena a Torino. Nelle loro missive, del resto, gli inviati sabaudi menzionano il timore diffuso che Eugenio Maurizio, sostenuto dalla moglie, faccia «qualche stregheria» per rendere sterile il matrimonio del duca di Savoia $^{71}$. La contessa di Soissons e la principessa di Carignano si sono guadagnate, infatti, la poco invidiabile fama di appassionate di sortilegi, tanto che la stessa Anna d'Austria si prende gioco del loro penchant per la magia ${ }^{72}$. Quanto a Luigi XIV, egli avrebbe accusato la principessa di Carignano di essere una «strega» e di voler gettare

${ }^{67}$ Sui principi di Savoia-Carignano e sul ramo Carignano-Soissons mancano studi complessivi. Cfr. Paola Bianchi, SAVOIA SOISSONS, Eugenio Maurizio di in "Dizionario Biografico" (treccani.it); Andrea Merlotti, SAVOIA CARIGNANO, Emanuele Filiberto Amedeo di in "Dizionario Biografico" (treccani.it); Idem, "La successione possibile: il principe di Carignano Emanuele Filiberto", in Torino 1675-1699: strategie e conflitti del barocco, ed. Giovanni Romano (Torino: CRT, 1993), 139-56. Sui princes étrangers alla corte di Francia, cfr. Guy Antonietti, "Les princes étrangers", in État et Société en France au XVIIe et XVIIIe siècles. Mélanges offerts à Yves Durand (Paris: 2000), 33-59; Simon Hodson, "Princes étrangers at the French Court in Seventeenth Century: the Grimaldi, the La Tour d'Auvergne and the La Trémoille", The Court Historian 1 (1998): 24-28. Sui principi del Sangue alla corte di Torino, cfr. Andrea Merlotti e Isabella Massabò Ricci, "In attesa del duca: reggenza e principi del sangue nella Torino di Maria Giovanna Battista", in Torino 1675-1699, 121-74.

${ }^{68}$ Lettere Ministri, Francia, m. 72: il marchese Pallavicino a Madama Reale, 15 dicembre 1662.

${ }^{69}$ Ibidem, Ghiron Villa a Madama Reale, 22 novembre 1662.

${ }^{70}$ Lettere Principi forestieri, m. 39, novembre 1662. Si veda anche la lettera dell'11 dicembre 1662: «'ai peur que l'on n'ait bien expliqué à S.A.R. mon neveu touchant celui qui épousera ma fille en son nom et les inconvénients qu'on peut appréhender, dans le moment même que se fera le mariage».

${ }^{71}$ Lettere Ministri, Francia, m. 73: Ghiron Villa a Carlo Emanuele, 15 dicembre 1662.

72 Ibidem: «La Regina Madre lunedì scorso che la signora principessa di Carignano fu al Louvre, li disse che si rallegrava che andasse aumentando in scienza, a segno che di presente fosse riputata per intendente di magia». 
una maledizione sui due sposi ${ }^{73}$. Sebbene tali accuse possano far sorridere, è interessante rilevare che, diversi anni dopo, proprio Olimpia Mancini sarà costretta ad abbandonare la Francia, in seguito al suo coinvolgimento nel celebre affaire des poisons ${ }^{74}$. Anche la stesura del contratto nuziale è segnata da un'accesa diatriba, che Margherita cerca, con scarsa fortuna, di dirimere. Luigi XIV rifiuta di designare Carlo Emanuele come Altezza, appellandosi all'antica tradizione di non usare titoli nei contratti firmati dal sovrano. Margherita propone di ultimare a Torino la stesura del contratto, in modo che la presenza del re non sia d'ostacolo ${ }^{75}$. Temendo che le difficoltà sollevate dal duca di Savoia possano ritardare il matrimonio o addirittura impedirne la conclusione, ella interviene presso Cristina, ricordandole che il re è pur sempre disposto a trattare Carlo Emanuele alla stregua di un membro della sua famiglia:

S.M. dit que dans votre contrat on ne parla point d'Altesse à feu Monsieur de Savoie, lequel ne le contestoit pas pour les mêmes raisons. Qu'aujourd'hui ce n'est point l'usage de France et cela n'a pas tort ni offense. Le Roi ne le conteste point mais l'usage n'est point en France de traiter d'Altesse dans les contrats quand le Roi les fait. Pour ceux que le Roi a fait et signés, où il y avoit des titres, il dit que l'on sait bien que lorsque le cardinal Mazarin faisoit tout qu'il lui laissoit tout faire, et que depuis sa mort il avoit résolu de remettre les choses dans les règles du temps d'Henri Quatre. Et pour moi, je sais qu'en faisant les contrats comme on l'a fait à Monsieur, que cela ne fait nul préjudice, au contraire, quand on dira que le Roi l'a traité comme les Enfants de France que cela ne sera qu'avantageux ${ }^{76}$.

Cristina e Carlo Emanuele si rassegnano male a quella che considerano come una cocente umiliazione. Probabilmente per questo motivo, Margherita passa bruscamente, nei confronti di Madama Reale, dal familiare ma soeur, senza ulteriori titoli, al più deferente Madame, accompagnato dalla formula Votre Altesse Royale: un'abile mossa per lusingare la duchessa di Savoia e consolarla dell'affronto ricevuto.

Tre giorni appena dopo le nozze al Louvre, Francesca Maddalena d'Orléans parte da Fontainebleau, per schivare le quérelles sul suo rango a corte, in particolare gli intrighi di Enrichetta Anna: quest'ultima ha infatti insistito - inutilmente - presso il re perché la giovane duchessa di Savoia la visitasse al Palais Royal prima di partire, ciò che le avrebbe permesso di rimarcare la propria precedenza sulla figlia di Gastone ${ }^{77}$.

${ }^{73}$ Ibidem, m. 72, il marchese Pallavicino a Madama Reale, 15 dicembre 1662: «La s. principessa di Carignano mi fece delle doglianze estreme, dicendomi che Sua Maestà gli aveva detto che era una strega et per questo V.A.R. e S.A.R. non la volevano in Piemonte [...] Ella non può credere che l'abbino in un concetto tanto pregiudiciale alla sua reputatione, che è che ella abbi pensato di fare qualche sortilegio a S.A.R. a fini che non abbia figlioli».

${ }^{74}$ Su Olimpia Mancini, cfr. Blythe A. Raviola, "Per il profilo di una Mazarinette. Olimpia Mancini di Savoia-Soissons", Cheiron 1 (2017): 100-127.

${ }^{75}$ Lettere Principi forestieri, m. 30, Margherita a Madama Reale, 14 gennaio 1663: «En cas que l'on ne pût surmonter les difficultés du costé du Roi, que l'on ne signe que les articles lesquels, comme vous savez, le sont déjà, et que l'on achève le mariage et que l'on fít le contrat à Turin, car je crois que les articles signés suffisent pour ce qui est dedans».

${ }^{76}$ Lettere Principi forestieri, m. 39, dicembre 1662.

${ }^{77}$ Lettere Ministri, Francia, m. 73: Ghiron Villa a Madama Reale, 17 gennaio 1663. 
4.

Se, nella competizione per la mano di Mademoiselle d'Orléans, il duca di Savoia ha dovuto cedere il passo ai Medici, l'unione con Mademoiselle di Valois rappresenta anch'essa una sorta di declassamento per Carlo Emanuele. Quest'ultimo, infatti, è il primo duca di Savoia, dopo Filippo II e la cugina Iolanda, a non sposare la figlia dell'imperatore o di un $\mathrm{re}^{78}$. Inoltre, Francesca Maddalena è frutto di un matrimonio dalla legittimità controversa; suo padre è di fatto morto in esilio, lontano dalla corte, e sua madre proviene da una dinastia di secondo piano in Europa, che attraversa peraltro una gravissima crisi politica e militare. Nei rapporti con Madama Reale e con il genero, Margherita tenta di compensare sul piano emotivo la propria debolezza sul piano cerimoniale, ricorrendo al registro dell'affettività. Le sue lettere rappresentano un vero e proprio pladoyer per guadagnarsi le grazie di Madama Reale, esplicitamente riconosciuta, tanto da Margherita quanto da Mademoiselle di Valois, come l'artefice del matrimonio sabaudo: «Je vous dirai», scrive Margherita a Cristina, «que, vous donnant une autre moi-même, ma joie sera continuellement à vous donner des marques pour elle et pour moi de la recognoissance que nous en aurons à jamais ${ }^{79}{ }$. La vedova di Gastone esprime nei termini più formali il debito della figlia verso Madama Reale:

Je vous réponds qu'elle n'a autre pensée ni n'aura jamais autres désirs que de suivre vos volontés, et qu'elle connoit fort bien les obligations qu'elle vous a et les ressent avec la dernière tendresse. Et toutes ses prières ne sont que présentement pour qu'elle soit assez heureuse pour que bientôt elle vous fasse assurée par ses soumissions et respects comme elle met tout son bonheur à suivre vos volontés et à vous donner des marques de son amour pour vous ${ }^{80}$.

Margherita non esita a definire Francesca Maddalena «notre fille», attribuendo a Cristina una maternità simbolica nei confronti della giovane duchessa; parallelamente, ella professa per Carlo Emanuele un affetto tutto materno: «Je ne peux m'empecher de vous faire cognoistre que j'ai pour V.A.R. toute la tendresse et l'amour qu'une mère peut avoir pour son fils ${ }^{81} \gg$. Nella sua prima lettera alla suocera, Francesca Maddalena si esprime sullo stesso tono della madre:

Comme je ne souhaite rien tant au monde que de donner des marques de ma reconnaissance à V.A.R., je ne saurois voir approcher le temps qui peut m'en donner les moyens sans en ressentir une extrême joie: celle que j'aurai de me voir auprès d'elle ne se peut exprimer, et le plaisir que je prendrai d'y vivre dans le dernier respect et une

\footnotetext{
${ }^{78}$ Andrea Merlotti, "Politique dynastique et alliances matrimoniales de la Maison de Savoie au XVIIe siècle", Revue Dix-septième siècle 61 (2009): 239-255.

${ }^{79}$ Lettere Principi forestieri, m. 39: 10 novembre 1662.

${ }^{80}$ Ibidem.

${ }^{81}$ Lettere Principi forestieri, m. 39, 13 dicembre 1662.
} 
entière dépendance. Je dois cela à V.A.R. et je serais la plus ingrate du monde si j’y manquais en la moindre chose ${ }^{82}$.

Anche nella scelta delle persone che devono accompagnare la sposa, una questione sovente all'origine di malumori, Margherita manifesta la propria deferenza verso Madama Reale. Pur selezionando accuratamente gli accompagnatori della figlia, ella protesta di rimettersi alla volontà di Cristina:

Mon dessein éstoit de vous demander le nombre des personnes que vous désiriez, mais M. votre ambassadeur m'a pressée de votre part d'envoyer un mémoire, ainsi je vous l'envoie, vous conjurant d'en user comme vous le trouverez plus à propos, puisque nos intérêts ne sont qu'un [...] Je vous conjure derechef qu'il vous plaise d'y retrancher et d'y écrire tout comme il vous plaira ${ }^{83}$.

Tuttavia, Margherita tenta di imporre comunque le proprie preferenze, soprattutto per quanto riguarda la governante di Francesca Maddalena, Claude Faye d'Espesses, contessa di Langeron. Margherita insiste affinché la contessa, molto legata a Mademoiselle di Valois, possa rimanere a Torino più a lungo degli altri:

Il serait très à propos que la comtesse de Langeron, qui mènera ma fille, demeure quelque temps auprès d'elle pour y continuer, autant que vous le trouverez nécessaire, ce qu'elle y a réussi jusqu'ici très fidèlement. Quand je pense que ma fille n'a que quatorze ans, je me trouve bien persuadée qu'elle a besoin encore d'une personne qui puisse l'advertir des choses que l'on ne peut pas encore savoir à son âge ${ }^{84}$.

Un'identica richiesta è presentata da Margherita in favore del confessore della giovane duchessa, Le Cointe, già rettore del collegio gesuita di Blois. Carlo Emanuele è molto diffidente nei confronti dei gesuiti, ma Margherita insiste con Madama Reale:

Comme ma fille de Valois a grande confiance en lui, qui est l'unique à qui elle s'est toujours confessée, et comme elle va changer de condition en un âge à lequel pour sa conscience elle doit être sagement instruite de choses qui demandent une grande confiance de sa part et beaucoup de discrétion de la part du confesseur: je serois bien navrée qu'elle aussi qu'elle fût obligée de le changer, parce que cela serait une contrainte et c'est une chose qu'on laisse toujours à la volonté et au choix de la fille dans tous les mariages ${ }^{85}$.

82 AsTo, Lettere Duchi e Sovrani, m. 66: Mademoiselle di Valois a Madama Reale, novembre 1662.

${ }^{83}$ Lettere Principi forestieri, m. 39, 10 novembre 1662.

${ }^{84}$ Ibidem., novembre 1662.

${ }^{85}$ Ibidem, 13 dicembre 1662. Si veda anche la lettera del 19 dicembre: «C'est celui qu'elle a toujours eu depuis qu'elle a été en âge d'en avoir un. Et je vous supplie de considérer que, l'ayant depuis qu'elle a été en âge de se confesser, qu'il lui serait pénible de le lui ôter. Néanmoins, comme je ne désire que toutes les choses que vous souhaitez, mandez-moi vos volontés». 
Il 23 marzo 1663, Francesca Maddalena arriva a Lione, evitando di passare per Grenoble, il cui Parlamento si è rifiutato di rendere omaggio al duca di Savoia designandolo col titolo di Altezza Reale ${ }^{86}$. Carlo Emanuele attende la sposa a Chambéry, dove riceve una lettera del confessore della duchessa, che conferma i suoi sospetti. Il duca di Savoia scrive a Madama Reale che il gesuita gli ha tenuto «un discours fort ambigu qui ne me plait pas: il est un homme fort intrigant, il a formé une cabale avec Madame de Langeron et il ne permet à personne de parler à Madame» ${ }^{87}$.

Di conseguenza, nonostante le proteste di Margherita, ancor prima di incontrare la sua sposa, Carlo Emanuele ordina che il sacerdote sia immediatamente rimpatriato. Margherita invia allora a sua figlia il curato della chiesa di Saint-Saveur a Blois, che ha assistito Gastone nei suoi ultimi istanti: «Comme S.A.R. a voulu un autre qu'un jésuite pour confesseur de ma fille», ella scrive a Cristina, «j'envoie celui qui a assisté feu Monsieur à la mort, qui est un homme savant et très homme de bien, afin que S.A.R. et vous, Madame, voyiez que je ferai toujours tout ce qui dépendra de moi pour votre satisfaction $\rangle^{88}$.

I due sposi si incontrano il 2 aprile ad Annecy, dove ricevono la benedizione nuziale dal vescovo di Ginevra; cinque giorni più tardi ha luogo l'ingresso solenne a Chambéry. In una lettera a Madama Reale, Ghiron Villa rileva che il matrimonio è stato consumato "con gioia e soddisfazione reciproca ${ }^{89}$ 》. Quanto a Carlo Emanuele, egli sembra gradire la giovanissima moglie, che ha appena compiuto quindici anni: «Enfin, ma chère Maman», egli scrive a Cristina, «je vous parle franchement, je vous ai bien d'obligation de m'avoir procuré le bonheur d'avoir votre chère nièce ${ }^{90} \%$. Margherita si felicita con Madama Reale nei termini consueti, rimarcando una volta di più la propria riconoscenza, al punto da insistere perché la duchessa non si spinga fino a Rivoli per accogliere la nuora ${ }^{91}$. Ma Carlo Emanuele rimane poco propenso ad accogliere le richieste della suocera, che insiste ancora perché Madame di Langeron possa restare accanto a sua figlia: il duca la rinvia immediatamente a Parigi, senza permetterle di raggiungere Torino.

${ }^{86}$ Lettere Principi forestieri, m. 39: Margherita a Madama Reale, 26 febbraio 1663; Matrimoni, m. 30, fasc. 5: Ghiron Villa a Carlo Emanuele, 6 marzo 1663.

${ }^{87}$ Lettere Duchi e Sovrani, m. 64: Carlo Emanuele a Madama Reale, 27 marzo 1663. La lettera del gesuita al duca di Savoia è andata dispersa; ci resta quella che il padre Le Cointe ha scritto per presentarsi a Madama Reale, durante la sosta a Langeron: Lettere di particolari, C, m. 87: 17 marzo 1663.

${ }^{88}$ Lettere Principi forestieri, m. 39, aprile 1663.

${ }^{89}$ Lettere Ministri, Francia, m. 73: Ghiron Villa a Madama Reale, 6 aprile 1663.

${ }^{90}$ Lettere Duchi e Sovrani, m. 64: Carlo Emanuele a Madama Reale, s. d. [aprile 1663].

${ }^{91}$ Lettere Principi forestieri, m. 39, Margherita a Madama Reale, 13 aprile 1663: «Je crois que V.A.R. saura comme ma fille a vu S.A.R. et qu'ils paroissent fort contents l'un de l'autre. J'espère que cela augmentera toujours et qu'il donnera à V.A.R. sujet de lui continuer votre amitié. Pour moi, Madame, tout ce que je pourrois faire est infinitement au-dessous de l'affection que vous m'avez fait paroistre, ma je vous supplie de croire que je n'épargnerai jamais rien pour le moindre de vos intérêts. Dans le souci que $j$ 'ai pour votre conservation, j'ose vous supplier de ne point partir de Turin pour aller audevant de ma fille pendant que le temps n'est pas encore trop bon». 
Dopo una sosta all'abbazia della Novalesa, dove li accoglie Emanuele Filiberto di Savoia-Carignano, i duchi di Savoia si dirigono a Rivoli, dove incontrano Cristina, prima dell'entrata solenne nella capitale il 14 maggio $1663^{92}$.

Nei mesi seguenti, Margherita continua ad appellarsi a Madama Reale, affinché sia di guida a Francesca-Maddalena:

Je demande à V.A.R. que vous lui disiez librement tout ce qu'elle doit faire, car je vous assure que si elle y manque c'est sans dessein: mais, au nom de Dieu, instruisez-la à tous ses devoirs, et considérez son âge. Et je vous demande de m'escrire franchement si vous êtes contente de notre fille ${ }^{93}$.

Margherita tenta, inizialmente, di esercitare qualche influenza sul genero, per esempio nella scelta di un primo segretario di Stato: temendo che le sue lettere siano aperte prima di giungere a Torino, la principessa ricorre a una scrittura in codice, nella quale i nomi sono sostituiti da cifre ${ }^{94}$. Ella garantisce a Madama Reale che «si vous en fiez en moi, je lui en donnerais un qui lui sera agréable et qui aura les qualités nécessaires pour cet emploi ${ }^{95}$ ». Tuttavia, né Cristina né Carlo Emanuele sembrano prendere in considerazione la sua offerta: la carica di primo segretario è affidata infatti a Carlo Vittorio Giuseppe Carron di San Tommaso, che affianca suo padre Francesco Guglielmo ${ }^{96}$.

Margherita cerca di intromettersi ugualmente nel ménage dei due sposi, preoccupandosi di chi potrebbe minacciarne l'armonia. Avendo saputo che JeanneMarie di Trécesson è rimasta a corte nonostante il suo matrimonio e le nozze di Carlo Emanuele, ella prega Madama Reale di allontanarla:

J'ai pris la liberté d'écrire à S. A.R. sur un bruit qui court ici que je ne crois point, mais, éstant obligée de prendre part à tout ce qui le regarde, je manqueroit de ne pas l'advertir de qui pouvoit diminuer de la sage conduite que tout le monde dit qu'il a. Ce bruit est que l'on dit qu'il a le dessein de continuer ces entretiens qu'il a eu si longtemps avec cette femme, et même pour cela qu'il veut la retenir à Turin. Cela feroit

92 Matrimoni, m. 30, fasc. 7: Relationi delle differenti solennità cerimoniali osservati in occasione del matrimonio del duca Carlo Emanuele II. Cfr. Giuliano Ferretti, L'entrée de Charles-Emmanuel II et Françoise d'Orléans à Turin (1663), in Becchia, Édifier l'État, 123-146; Idem, "Mariages princiers dans la Maison de Savoie. Ménestrier et les entrées à Annecy et Chambéry (1663)", in Claude-Francois Ménestrier. Les Jésuites et le monde des images, dir. Gérard Sabatier (Grenoble: PUG, 2009), 263-88 ; Paola Bianchi, "Politica matrimoniale e rituali fra Cinque e Settecento", in Le strategie dell'apparenza. Cerimoniali, politica e società alla corte dei Savoia in età moderna, eds. Paola Bianchi e Andrea Merlotti (Torino: Zamorani, 2010), 53-56.

93 Ibidem, 8 giugno 1663.

${ }_{94}$ Ibidem, Margherita di Lorena a Ghiron Villa, 2 gennaio 1663.

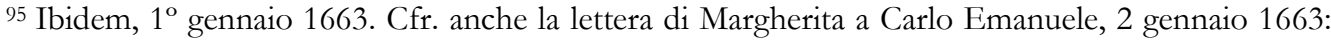
«Je crois devoir encore vous faire cognoistre que si vous avez agréable de vous confier à moi pour le choix d'un secrétaire, que vous en serez content».

${ }^{96}$ Sui segretari di Stato nel ducato sabaudo di età moderna, cfr. Claudio Rosso, Una burocrażia di antico regime: $i$ segretari di Stato dei duchi di Savoia, 1559-1637 (Torino: Deputazione Subalpina di Storia Patria, 1992). 
assurément tort à la sage conduite que l'on croit qu'il a, ainsi je vous supplie de le lui faire cognoistre ${ }^{97}$.

Merita notare che il tutore di Jeanne-Marie, Anne de Brûlon, è molto vicino ai Carignano-Soissons: non è escluso che tale prossimità alimenti l'inquietudine di Margherita nei confronti della favorita di Carlo Emanuele ${ }^{98}$. Anche in questo caso, però, le sue preghiere non fanno breccia nell'animo di Madama Reale, che si guarda bene da intervenire presso il figlio per separarlo dalla marchesa di Cavour. Del resto, sia Cristina che Margherita hanno ben presto altre preoccupazioni: la prima vede la propria salute degradarsi velocemente, mentre la seconda si inquieta sempre più per la figlia.

Nel dicembre 1663, le infermità che già da qualche anno affliggono Cristina si aggravano bruscamente. Margherita ricorda a Francesca Maddalena il proprio debito verso la suocera, raccomandandole di assisterla amorevolmente:

Ma chère enfant, ce mot n'est que pour vous dire que je suis dans la dernière peine du mal de Madame Royale. Témoignez-lui bien cela de ma part et rendez-lui toutes les marques qu'une bonne fille doit à sa mère: vous ne sauriez jamais recognoistre les obligations que vous lui avez ${ }^{99}$.

Madama Reale muore a Torino il 26 dicembre. Nella lettera che Margherita indirizza a Carlo Emanuele si avverte la consapevolezza di aver perduto un'influente protettrice:

Mon cher fils, c'est avec la dernière douleur que j'ai appris par la vôtre la perte que nous avons faite de feu Madame Royale. V.A.R. me permettra bien que je parle ainsi, puisqu'elle sait bien les obligations que je lui avoit, qui est d'avoir contribué au bonheur inestimable de ma fille ${ }^{100}$.

Peraltro, Margherita deve far fronte alle umilianti critiche del duca di Savoia e dei suoi ministri: il medico Philibert Morisset, da lei fortemente raccomandato, è accusato di aver accelerato il decesso di Cristina e immediatamente rimpatriato. Mortificata, Margherita prega il genero di scegliere un altro medico francese per la moglie, la cui salute va rapidamente deteriorandosi ${ }^{101}$.

\footnotetext{
${ }^{97}$ Lettere Principi forestieri, m. 39, s. d. [gennaio 1663]. Si veda anche un’altra lettera senza data: «Je ne peux m'empêcher de vous dire qu'ayant appris que cette femme que S.A.R. a si longtemps aimée était à Turin, il est bien à craindre que cette inclination ne reprenne et que cela ne fasse quelque diminution à l'affection qu'il avoit pour sa femme. Ainsi il est de votre prudence de voir si on ne pourroit point obtenir de S.A.R. qu'il l'éloigne».

${ }^{98} \mathrm{Si}$ vedano le sue lettere a Cristina di Francia: Lettere di particolari, B, m. 125, 1658-1659.

${ }^{99}$ Lettere Principi forestieri, m. 39: Margherita a Francesca-Maddalena, 28 dicembre 1663.

100 Ibidem, 11 gennaio 1664.

101 Ibidem: «Je suis marrie du médecin Morisset, qui assurément avoit été choisi des premiers médecins du Roi et des Reines, et de la première école de ceux qui sont dans Paris. V.A.R. en a usé fort sagement, mais je la supplie d'être persuadé que s'il ne donne pas un médecin françois à ma fille qu'avant que vos médecins aient bien connu son tempérament, elle en souffrira pour sa santé». Su Philibert
} 
Non possiamo sapere se la scomparsa di Cristina abbia rappresentato un'autentica perdita per Francesca Maddalena o se, al contrario, la giovane duchessa si sia sentita libera da una suocera troppo ingombrante.

Prima del suo arrivo a Torino, gli inviati sabaudi avevano già notato che Mademoiselle di Valois era afflitta da febbri frequenti, che avevano perfino interrotto il suo ciclo mestruale ${ }^{102}$. Durante la cerimonia nuziale, la sposa, convalescente, si è sentita male ed è quasi svenuta; nel corso del viaggio verso la Savoia ha dovuto fermarsi a Langeron per recuperare le forze ${ }^{103}$. Margherita ha supplicato Madama Reale di trattenere a Torino il personale che ha seguito Francesca Maddalena da Parigi, in particolare Philibert Morisset ${ }^{104}$. Convalescente da un'indisposizione che ha fatto sperare in una gravidanza ${ }^{105}$, Francesca Maddalena è ben presto attanagliata da una febbre che cerca di nascondere il più a lungo possibile, per poter assistere la suocera agonizzante: la giovane duchessa muore a Torino il 14 gennaio 1664. La sua insistente richiesta di non essere sottoposta ad autopsia viene ignorata: i medici, si legge in una relazione dell'epoca, le trovano «una grandissima infiamatione di polmone, il fegato

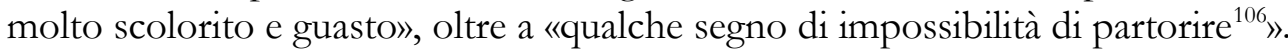

Lo stato lacunoso delle fonti non ci consente di leggere le lettere indirizzate da Margherita a Carlo Emanuele subito dopo il decesso di Francesca Maddalena: la corrispondenza, infatti, si interrompe bruscamente all'inizio di gennaio 1664, per riprendere nel giugno 1666, quando la principessa si felicita con il duca di Savoia, risposatosi con Maria-Giovanna Battista di Savoia-Nemours, per la nascita del principe di Piemonte ${ }^{107}$.

\section{RIFLESSIONI CONCLUSIVE}

È probabile che, aldilà del dolore per la perdita di una figlia, Margherita di Lorena abbia percepito la morte della duchessa di Savoia, incapace di fornire un erede alla dinastia sabauda, come un insuccesso personale, la tragica conclusione di

Morisset, cfr. Correspondance complète et autres écrits de Guy Patin, ed. Loïc Capron (Paris: Bibliothèque Interuniversitaire de Santé, 2018), //www.biusante.parisdescartes.fr/patin/?do=pg\&let=0737 (consultato il 18/02/2021).

102 Matrimoni, m. 30, fasc. 5: Ghiron Villa a Carlo Emanuele, 8 novembre 1662 e 26 febbraio 1663.

103 Lettere Ministri, Francia, m. 73: Ghiron Villa a Carlo Emanuele, 21 marzo 1663.

104 aprile 1663: «Je me trouve fort obligée de supplier V.A.R. de vouloir faire agréer à S.A.R. que l'apothicaire qui emmène ma fille demeure, car assurément le service seroit mal fait si le médecin et l'apothicaire n'éstoient pas françois»; ibidem, 13 aprile 1663: «Il faut que je vous supplie de ne point laisser revenir le médecin Morisset et de le retenir à quelque prix que ce soit, car vous n'en trouverez pas un semblable; l'apothicaire est absolument nécessaire, V.A.R. le retiendra s'il vous plait, pour ma fille, je l'en supplie».

105 Ibidem, Margherita a Madama Reale, 21 ottobre [1663].

106 Torino, Biblioteca Reale, Misc. 168: Breve racconto di molte particolarità seguite nella morte di fu M.R. et anche in quella di fu Madama la Duchessa Reale, occorse in dieci nove giorni soli distanti, f. 23v. Il testo è attribuibile a un domestico della contessa Costa di Polonghera, dama d'onore di Francesca Maddalena.

${ }^{107}$ Lettere Principi forestieri, m. 30, 4 giugno 1666. 
un'alleanza da lei ardentemente sostenuta: «Vous jugerez da ma joie, Madame», scrive Margherita a Cristina, dopo l'incontro tra quest'ultima e Francesca Maddalena, «par l'ardente passion que j'ai eu de voir ce mariage achevé ${ }^{108}$ ». Tuttavia, il gioco diplomatico che la vedova di Gastone ha condotto dietro le quinte, nonostante le manifeste ostilità con cui ha dovuto scontrarsi, può senza dubbio essere considerato un successo. Ella riesce a sfruttare il linguaggio dell'amitié e della tendresse per ribadire la propria comunione di interessi con Cristina e con il duca di Savoia; inoltre, non esita ad appoggiarsi alla rete clientelare di Madama Reale, fino ad integrarla, autoproclamarsi una protégée della duchessa di Savoia. Se è vero che, sul lungo termine, i tentativi della duchessa d'Orléans per accreditarsi come intermediaria presso la corte di Torino falliscono, a causa del crescente isolamento a cui è condannata a Parigi, le sue lettere, nella congiuntura del matrimonio sabaudo, mettono in luce le opportunità che una società di corte multicentrica offre a una principessa dallo status elevato, ma controverso, di esercitare un'influenza informale sulle strategie matrimoniali della dinastia regnante.

108 Ibidem, 31 maggio 1663. 


\section{BIBLIOGRAFIA}

Marcella Aglietti e José A. López Anguita, eds., Élites internazionali e reti di potere (Pisa: Pisa University Press, 2016.

Nadine Akkerman e Birgit Houben, eds., The Politics of Female Households: Ladies-inwaiting across Early Modern Europe (Leiden, Brill, 2014).

Bernard Allorent, La fortune de la Grande Mademoiselle (Paris: Champion, 2019).

Antonio Álvarez-Ossorio, Cinzia Cremonini e Elena Riva, eds., The Transition in Europe between XVII ${ }^{\text {th }}$ and XVIII th centuries. Perspectives and Case Studies, (Milano: Franco Angeli, 2016).

Stefano Andretta, Stéphane Peguinot e Marie-Karine Schaub, eds., Paroles de négociateurs. L'entretien dans la pratique diplomatique de la fin du Moyen Age à la fin du XIXe siècle (Roma: École Française, 2010).

Stefano Andretta, Lucien Bély e Geraud Poumarède, dir., Esperienza e diplomazia. Saperi, pratiche culturali e azione diplomatica nell'Età moderna (secc. XV-XVIII) (Roma: Viella, 2020).

Alain Becchia e Florine Vital-Durand, eds. Édifier l'État: politique et culture en Savoie au temps de Christine de France (Chambéry: USMB, 2014).

Lucien Bély, La société des princes, XVI'-XVIII siècle, Paris, Fayard, 1999.

—, Les Secrets de Louis XIV. Mystères d'État et pouvoir absolu (Paris: Tallandier, 2015).

-, "Christine de France, duchesse de Savoie, et la politique française au temps de Mazarin", Revue Dix-Septième Siècle 262 (2014): 21-29.

_, Espions et ambassadeurs au temps de Louis XIV (Paris: Fayard, 1990).

Marco Bellabarba e Andrea Merlotti, ed., Stato Sabaudo e Sacro Romano Impero (Bologna: Il Mulino, 2014).

Paola Bianchi, SAVOIA SOISSONS, Eugenio Maurizio di in "Dizionario Biografico" (treccani.it)

Paola Bianchi e Andrea Merlotti, eds., L'affermarsi della corte sabauda. Dinastie, poteri, élites in Piemonte e Savoia fra tardo medioevo e prima età moderna, (Torino: Zamorani, 2006).

—, Le strategie dell'apparenza. Cerimoniali, politica e società alla corte dei Savoia in età moderna, (Torino: Zamorani, 2010). 
Anna Blum, La diplomatie de la France en Italie du Nord au temps de Richelieu et de Mazarin (Paris: Garnier, 2014).

Mathilde Bombart e Éric Méchouan, eds., Politiques de l'épistolaire au XVII siècle. Autour $d u$ Recueil Faret (Paris: Garnier, 2019).

Michel Bougain, "La comédie de Lyon: relecture d'un mythe historique", Revue Dixseptième Siècle 274 (2017): 129-59.

Clarissa Campbell-Orr, dir., Queenship in Europe 1660-1815, (Cambridge: CUP, 2004).

Diana Carrió-Invernizzi, "A New Diplomatic History and the Networks of Spanish Diplomacy in the Baroque Era," The International History Review 36 (2014): 603618.

Vittorio Castronovo in Diquionario Biografico degli Italiani, Roma, Treccani, vol. 20, 1977, CARLO EMANUELE II, duca di Savoia in "Dizionario Biografico" (treccani.it)

Alessandro Celi e Mathew Vester, eds., Tra Francia e Spagna. Reti diplomatiche, territori e culture nei domini sabaudi fra Tre e Settecento (Roma: Carocci, 2017).

Adolphe Chéruel, Mémoires sur la vie publique et privée de Fouquet (Paris: Charpentier, 1862)

Gaudenzio Claretta, Storia del regno di Carlo Emanuele II (Genova: 1877).

Jean-Marie Constant, Gaston d'Orléans, prince de la liberté (Paris: Perrin, 2013).

Correspondance complète et autres écrits de Guy Patin, ed. Loïc Capron (Paris: Bibliothèque Interuniversitaire de Santé, 2018), //www.biusante.parisdescartes.fr/patin/?d $\underline{\mathrm{o}=\text { pg\&let }=0737}$

Dejanirah Couto e Stéphane Péguinot, dir., Les langues de la négociation. Approches bistoriennes (Rennes: PUR, 2017).

Benedetta Craveri, L’âge de la conversation (Paris: Gallimard, 2002).

Lucien De Warren, "Marguerite de Lorraine, duchesse d'Orléans", Bulletin de la Société Philomatique Vosgienne (1882-1883): 137-175.

Dorsale catholique, Jansénisme, Dévotions: XVIe-XVIII siècles, Mythe, réalité, actualité historiographique (Paris: Riveneuve, 2014).

État et Société en France au XVIIe et XVIIIe siècles. Mélanges offerts à Yves Durand (Paris: 2000). 
Indravati Felicité, dir., L'identité du diplomate (Moyen Âge-XIX siècle). Métier ou noble loisir? (Paris: Garnier, 2020).

Giuliano Ferretti, dir., Les États de Savoie, du duché à l'Unité d'Italie (1416-1861) (Paris: Garnier, 2020), 261-283.

-, ed., L'État, la cour et la ville. Le duché de Savoie au temps de Victor-Amédée Ier et de Christine de France, 1618-1663 (Paris: Classiques Garnier, 2018).

Jean-Louis Fournel e Matteo Residori, eds. Ambassades et ambassadeurs en Europe (XV'XVII siècles) (Genève: Droz, 2020).

Pierre Gatulle, Gaston d'Orléans, entre mécénat et impatience du pouvoir (Seyssel: Champ Vallon, 2012).

Rubén Gonzalez Cuerva e Alexander Koller, dir., A Europe of Courts, a Europe of Factions: Political Groups at Early Modern Centres of Power (1550-1700), (Brill, 2017).

Dena Goodman, "Public Sphere and Private Life: Toward a Synthesis of Current Historiographical Approaches to the Old Regime," History and Theory 31 (1992): $1-20$.

Graham C. Gibbs, Robert Oresko e H. M. Scott, eds, Royal and Republican Sovereignty in Early Modern Europe, (Cambridge: CUP, 1997).

Simon Hodson, "Princes étrangers at the French Court in Seventeenth Century: the Grimaldi, the La Tour d'Auvergne and the La Trémoille", The Court Historian 1 (1998): 24-28.

Alain Hugon, Au service du Roi Catholique. Honorables ambassadeurs et divins espions (Madrid: Casa de Vélazquez, 2004).

Vincenzo Lagioia, La verità delle cose. Margherita Luisa d'Orléans: donna e sovrana d'ancien régime (Roma: Edizioni di Storia e Letteratura, 2015.

La Savoie et ses voisins dans l'histoire de l'Europe (Annecy: Académies Florimontane et Salésienne, 2010).

Nicolas Marquis, "La résilience comme attitude face au malheur", SociologieS, Théories et recherches (2018), http://journals.openedition.org/sociologies/6633

Mémoires de Mademoiselle de Montpensier, ed. Adolphe Chéruel, Paris, Charpentier, 18581859. 
Pierpaolo Merlin, "Vassal de la France ou État de Souverain? Le duché de Savoie et les traités de Westphalie (1641-1648)", Revue Dix-Septième Siècle 262 (2014): 3142.

Andrea Merlotti, SAVOIA CARIGNANO, Emanuele Filiberto Amedeo di in "Dizionario Biografico" (treccani.it)

Anne Motta, Noblesse et pouvoir princier dans la Lorraine ducale, 1624-1737 (Paris: Garnier, 2016).

Frank Muller e Heidi Mehrkens, eds. Royal Heirs and the Uses of Soft Power in Nineteenth-Century Europe (Palgrave, 2016).

Joseph S. Nye, "Public Diplomacy and Soft Power," The Annal of American Academy of Political and Social Science 616 (2008): 94-109.

Toby Osborne, "The surrogate war between the Savoys and the Medici: Sovereignty and Precedence in Early Modern Italy", The International History Review 29 (2007): 1-21.

-, Dynasty and Diplomacy in the Court of Savoy: Political Culture and the Thirty Years War (Cambridge: CUP, 2000).

Andrea Pennini, "Con la massima diligentia possible". Diplomazia e politica estera sabauda nel primo Seicento (Roma: Carocci, 2015).

Giorgio Peyron, Jeanne-Marie de Trécesson, marchesa di Cavour (Savigliano: L'Artistica Savigliano, 1990.

Vincent Pitts, La Grande Mademoiselle at the court of France: 1627-1693 (London: The Johns Hopkins University Press, 2000).

Politics and Diplomacy in Early Modern Italy. The Structure of Diplomatic Practice, 1450-1800 (Cambridge: CUP, 2000).

Géraud Poumarède, "Deux têtes pour une couronne: la rivalité entre la Savoie et Venise pour le titre royal de Chypre au temps de Christine de France", Revue Dix-septième siècle 262 (2014): 53-64.

-, "Mazarin marieur de l'Europe. Stratégies familiales, enjeux dynastiques et géopolitique au milieu du XVIIe siècle”, Revue Dix-Septième Siècle 243 (2009): 201-218. 
Blythe A. Raviola, "Per il profilo di una Mazarinette. Olimpia Mancini di SavoiaSoissons", Cheiron 1 (2017): 100-127.

Blythe A. Raviola e Clelia Arnaldi, eds., Maria-Giovanna Battista di Savoia-Nemours (Torino: Allemandi, 2017).

Réseaux de femmes, femmes en réseaux (XVI'-XVIII siècles) (Bordeaux: Presses Universitaires de Bordeaux, 2018).

Ercole Ricotti, Storia della monarchia piemontese (Firenze: Barbera, 1861-1869).

Giovanni Romano, ed., Torino 1675-1699: strategie e conflitti del barocco, (Torino: CRT, 1993).

Claudio Rosso, Una burocrazia di antico regime: $i$ segretari di Stato dei duchi di Savoia, 1559 1637 (Torino: Deputazione Subalpina di Storia Patria, 1992).

Gérard Sabatier, dir., Claude-François Ménestrier. Les Jésuites et le monde des images, (Grenoble: PUG, 2009).

L. de Saint-Simon, Mémoires, ed. Arthur de Boislisle, Paris, Hachette, 1879-1931.

Daniel Serré, "Mazarin et la Comédie de Lyon: au-delà de la légende", Revue Dixseptième Siècle 231 (2006): 327-340.

Tracey A. Sowerby e Jan Hennings, eds., Pratices of Diplomacy in the Early Modern World (Routledge, 2017).

Jonathan Spangler, "Bridging the Gaps: The Household Account Books of Marguerite de Lorraine, Duchesse d'Orléans", Annales de l'Est 2 (2017): 69-86.

Giora Sternberg, "Epistolary Ceremonial: Corresponding Status at the Time of Louis XIV”, Past and Present 204 (2009): 33-88.

Christopher Storrs, War, Diplomacy and the Rise of Savoy, 1690-1720 (Cambridge: CUP, 1999).

Anne Twomey, "The Exercise of Soft Power by Female Monarchs in the United Kingdom,”, Royal Studies Journal 7 (2020): 31-48.

Maaike Van Berkel e Jeroen Duindam, eds, Prince, Pen and Sword: Eurasian Perspectives, (Leiden-Boston: Brill, 2018). 
Franca Varallo, ed., In assenza del Re. Le Reggenti nei secoli XVI-XVII (Piemonte ed Europa) (Firenze: Olschki, 2008).

Sophie Vergnes, Les Frondeuses. Une révolte au féminin (1643-1661) (Seyssel: Champ Vallon, 2013.

Mathew Vester, ed., Sabaudian Studies. Political Culture, Dynasty and Territory, 1400-1700 (Truman State University Press, 2013).

—, Jacques de Savoie-Nemours. L'apanage du Genevois au cour de la puissance dynastique savoyarde an XVI siècle (Genève: Droz, 2008).

Recibido: 16 de abril de 2021

Aceptado: 26 de mayo de 2021 